\title{
DELTA-SHOCKS, THE RANKINE-HUGONIOT CONDITIONS, AND SINGULAR SUPERPOSITION OF DISTRIBUTIONS
}

\author{
V. M. SHELKOVICH
}

\begin{abstract}
The problem of defining $\delta$-shock wave type solutions of hyperbolic systems of conservation laws in connection with the constructing singular superpositions (products) of distributions is studied. We illustrate this problem by constructing $\delta$-shock wave type solutions for two systems. One of them,

$$
u_{t}+f(u)-v_{x}=0, \quad v_{t}+g(u)_{x}=0,
$$
\end{abstract}

is a generalization of the well-known Keyfitz-Kranzer system, where $f(u)$ and $g(u)$ are polynomials of degree $n$ and $n+1$, respectively, $n$ is an even integer. The other one is the system

$$
u_{t}+f(u)_{x}=0, \quad v_{t}+v g(u)_{x}=0,
$$

where $f(u), g(u)$ are smooth functions. As far as we know, exact $\delta$-shock wave type solutions for the first system have never been constructed.

\section{INTRODUCTION}

1.1. Singular solutions to systems of conservation laws. Let us consider the hyperbolic system of conservation laws

$$
L_{1}[u, v]=u_{t}+(F(u, v))_{x}=0, \quad L_{2}[u, v]=v_{t}+(G(u, v))_{x}=0,
$$

where $F(u, v), G(u, v)$ are smooth functions, linear with respect to $v ; u=u(x, t), v=$ $v(x, t) \in \mathbb{R} ; x \in \mathbb{R}$. As is well known, even in the case of smooth (and, certainly, in the case of discontinuous) initial data $\left(u^{0}, v^{0}\right)$, this system may have discontinuous solutions. In this case, it is said that a pair $(u, v) \in L^{\infty}\left(\mathbb{R} \times(0, \infty) ; \mathbb{R}^{2}\right)$ is a generalized solution of the Cauchy problem (1.1) with the initial data $\left(u^{0}, v^{0}\right)$ if the integral identities

$$
\begin{aligned}
& \int_{0}^{\infty} \int\left(u \varphi_{t}+F(u, v) \varphi_{x}\right) d x d t+\int u^{0}(x) \varphi(x, 0) d x=0 \\
& \int_{0}^{\infty} \int\left(v \varphi_{t}+G(u, v) \varphi_{x}\right) d x d t+\int v^{0}(x) \varphi(x, 0) d x=0
\end{aligned}
$$

hold for all compactly supported test functions $\varphi(x, t) \in \mathcal{D}(\mathbb{R} \times[0, \infty))$, where $\int \cdot d x$ denotes an improper integral $\int_{-\infty}^{\infty} \cdot d x$.

It is well known [1], [10], [11], [12], [13], [14], [25] that there are "nonclassical" situations when the Riemann problem does not possess a weak $L^{\infty}$-solution except for some particular initial data. In contrast to the standard results of existence of weak solutions to strictly hyperbolic systems, here the linear component of the solution $v$ may contain Dirac measures and must be sought in the space of measures,

Date:

2000 Mathematics Subject Classification. Primary 35L65; Secondary 35L67, 76L05.

Key words and phrases. Hyperbolic systems of conservation laws, $\delta$-shock wave type solution, the Rankine-Hugoniot conditions, the weak asymptotics method, singular superpositions (products) of distributions.

The author was supported in part by DFG Project 436 RUS 113/593/3 and by Grant 02-0100483 of Russian Foundation for Basic Research. 
while the first component $u$ has bounded variation. In order to solve the Cauchy problem in this nonclassical situation, it is necessary to introduce new singularities called $\delta$-shocks, which are solutions of the hyperbolic system (1.1), such that the linear component of the solution can have the form $v(x, t)=V(x, t)+e(x, t) \delta(\Gamma)$, $\Gamma$ is a connected graph in the upper half-plane $\{(x, t): x \in \mathbb{R}, t \geq 0\}, V \in L^{\infty}(\mathbb{R} \times$ $(0, \infty) ; \mathbb{R}), e \in C^{1}(\Gamma)$.

Several approaches to constructing $\delta$-shock type solutions are known. An apparent difficulty in defining such solutions arises due to the fact that, to introduce a definition of the $\delta$-shock type solution, we need to define a singular superposition of distributions (for example, the product of the Heaviside function and the delta function). We also need to define in which sense a distributional solution satisfies nonlinear systems.

In particular, it is well known, that for some cases of system (1.1) the Cauchy problem with the initial data

$$
u^{0}(x)=u_{0}+u_{1} H(-x), \quad v^{0}(x)=v_{0}+v_{1} H(-x),
$$

where $u_{0}, u_{1}, v_{0}, v_{1}$ are constants and $H(\xi)$ is the Heaviside function, may admit a $\delta$-shock wave type solution, i.e., a generalized solution of the form

$$
\begin{aligned}
& u(x, t)=u_{0}+u_{1} H(-x+c t), \\
& v(x, t)=v_{0}+v_{1} H(-x+c t)+e(t) \delta(-x+c t),
\end{aligned}
$$

where $e(t)$ is a smooth function such that $e(0)=0$ and $\delta(\xi)$ is the Dirac delta function.

For example, in [11], in order to construct a $\delta$-shock wave type solution of the system

$$
L_{21}[u]=u_{t}+(f(u))_{x}=0, \quad L_{22}[u, v]=v_{t}+(g(u) v)_{x}=0,
$$

(here $F(u, v)=f(u), G(u, v)=v g(u))$ this system is reduced to a system of Hamilton-Jacobi equations, and then the Lax formula is used. In [10], a $\delta$-shock wave type solution of system (1.5) is constructed as self-similar viscosity limits. In [14], to construct a $\delta$-shock wave type solution of system (1.5) for the case $g(u)=f^{\prime}(u)$, the problem of multiplication of distributions is solved by using the definition of Volpert's averaged superposition [27]. In [20], a general framework for nonconservative product

$$
g(u) \frac{d u}{d x}
$$

was introduced, where $g: \mathbb{R}^{n} \rightarrow \mathbb{R}^{n}$ is locally bounded Borel function and $u$ : $(a, b) \rightarrow \mathbb{R}^{n}$ is a discontinuous function of bounded variation. In the framework of the approach [20] the Cauchy problems for nonlinear hyperbolic systems in nonconservative form can be considered [14], [15], [16]. Note that in [15], [16], for nonconservative systems the notion of generalized solution does depend on the specific family of paths, which can not be derived from the hyperbolic system only.

In [26], for the system

$$
u_{t}+\left(u^{2}\right)_{x}=0, \quad v_{t}+(u v)_{x}=0,
$$

(here $F(u, v)=u^{2}, G(u, v)=v u$ ) with the initial data (1.3), the $\delta$-shock wave type solution is defined as a measure-valued solution.

In $[13]$ for the system

$$
u_{t}+\left(u^{2}-v\right)_{x}=0, \quad v_{t}+\left(\frac{1}{3} u^{3}-u\right)_{x}=0
$$

(here $F(u, v)=u^{2}-v, G(u, v)=\frac{1}{3} u^{3}-u$ ) with the initial data (1.3) the $\delta$-shock wave type approximate solution was studied. But the notion of the exact $\delta$-shock 
solution has not been defined. In order to construct approximate solutions, the Colombeau theory approach, as well as the Dafermos-DiPerna regularization (under assumption that Dafermos profiles exist), and the box approximations are used. In [21] the existence of Dafermos profiles for singular shocks is proved. In [22], a class of problems for which the lowest-order asymptotic approximations to Dafermos profiles can be constructed is identified. System (1.8) is an example of a system satisfying general hypotheses of paper [22].

In [3], [4]- [9], [23], [24] a new approach to solving the problem of the propagation and interaction of singular fronts was developed. This approach was called the weak asymptotics method. The key role in this method is played by the definition of a weak asymptotic solution of the Cauchy problem, which admits passing to the limit in the weak sense as $\varepsilon \rightarrow 0$, where $\varepsilon$ is the regularization parameter. Using V. P. Maslov's idea, this method permits to derive the Rankine-Hugoniot conditions directly from the differential equations considered in the weak sense. V. P. Maslov's algebras of singularities are essential in our method [18], [19], [2]. By using the weak asymptotics method in above mentioned papers, the dynamics of propagation and interaction of different nonlinear waves (infinitely narrow $\delta$-solitons, shocks, $\delta$-shocks) of nonlinear equations and hyperbolic systems of conservation laws is studied. In the framework of the weak asymptotics method [7]- [9] new Definition 2.1, of a $\delta$-shocks type solution for (1.1) was introduced. This definition is a natural generalization of the usual system of integral identities (1.2).

1.2. Main results. In Sec. 2 we introduce the definition of a $\delta$-shock wave type solution for system (1.1), as well as the definition of a weak asymptotic solution, which is one of the most important notions in the weak asymptotics method. In this section we also derive $\delta$-shock Rankine-Hugoniot conditions. In order to construct a weak asymptotic solution of our problems, some weak asymptotics are constructed in Sec. 6.

In Sec. 3 we study the problem of propagation of a $\delta$-shock in the system

$$
\begin{aligned}
& L_{11}[u, v]=u_{t}+(f(u)-v)_{x}=0, \\
& L_{12}[u, v]=v_{t}+(g(u))_{x}=0,
\end{aligned}
$$

where

$$
f(u)=\sum_{k=0}^{n} A_{k} u^{k}, \quad A_{n} \neq 0, \quad g(u)=\sum_{k=0}^{n+1} B_{k} u^{k}, \quad B_{n+1} \neq 0,
$$

are polynomials, $n$ is an even number. The well known Keyfitz-Kranzer system (1.8) is a particular case of system (1.9). Thus we solve the Cauchy problem for system (1.9) with the $\delta$-shock front initial data

$$
\begin{aligned}
& u^{0}(x)=u_{0}^{0}(x)+u_{1}^{0}(x) H(-x), \\
& v^{0}(x)=v_{0}^{0}(x)+v_{1}^{0}(x) H(-x)+e^{0} \delta(-x),
\end{aligned}
$$

where $u_{k}^{0}(\mathrm{x}), v_{k}^{0}(x), \quad k=0,1$ are given smooth functions, $e^{0}$ is a given constant.

In Sec. 4 , the problem of propagation of the $\delta$-shock in system (1.5), solved in [6][9] is considered.

Remark 1.1. The Keyfitz-Kranzer system (1.8) and system (1.9) differ from system (1.5) and have a specific "strange" property. Although $\delta$-shock wave type solutions of the Cauchy problems (1.9), (1.11) and (1.5), (1.11) satisfy the same integral identity (2.1), in systems (1.9), (1.8) have no balance of singularities. If $(u, v)$ is a $\delta$-shock type solution (1.4) of system (1.8) then $u$ contains the Heaviside function $H$, and $v$ contains the Heaviside function $H$ and $\delta$-function (see (1.4). Thus, $u^{2}-v$ contains the distributions $H, \delta$, and $\frac{1}{3} u^{3}-u$ contains the distribution $H$. It is clear that 
the term $\left(u^{2}-v\right)_{x}$ contains $H, \delta, \delta^{\prime}$, while the term $u_{t}$ contains only $H$ and $\delta$. Analogously, the term $v_{t}$ contains $H, \delta, \delta^{\prime}$, but the term $\left(u^{3} / 3-u\right)_{x}$ contains only $H, \delta$. Seemingly, it is impossible to obtain $\delta$-shock type solutions for systems (1.8) and (1.9). Nevertheless, in Sec. 3, we prove that there are exact solutions of this type. First, $\delta$-shock wave type solutions for specific systems (1.8), (1.9) were constructed in [23] for piecewise constant initial data.

The problem of defining $\delta$-shock wave type solutions for the Cauchy problems (1.9), (1.11) and (1.5), (1.11) in connection with the construction of singular superpositions (products) of distributions is discussed in Sec. 5. We stress that the "right" singular superpositions of distributions (5.6)-(5.9) can be obtained only in the context of constructing weak asymptotic solutions to these Cauchy problems.

It remains to note that, since in the "specific" systems (1.9) and (1.8) there are no terms of the type of (1.6) (see (5.6), (5.7)), it is impossible to construct a $\delta$-shock wave type solution for them by using the nonconservative product [15], [16], [20].

1.3. The scheme of the weak asymptotics method. According to our method, we shall seek a $\delta$-shock wave type solution of the Cauchy problems (1.9), (1.11) and (1.5), (1.11) in the form

$$
\begin{aligned}
& u(x, t)=u_{0}(x, t)+u_{1}(x, t) H(-x+\phi(t)), \\
& v(x, t)=v_{0}(x, t)+v_{1}(x, t) H(-x+\phi(t))+e(t) \delta(-x+\phi(t)),
\end{aligned}
$$

where $u_{0}(x, t), u_{1}(x, t), v_{0}(x, t), v_{1}(x, t), e(t), \phi(t)$ are desired functions. This singular ansatz preserves the structure of the initial data (1.11). Within the framework of the weak asymptotics method, we find a $\delta$-shock wave type solution (1.12) as a weak limit

$$
u(x, t)=\lim _{\varepsilon \rightarrow+0} u(x, t, \varepsilon), \quad v(x, t)=\lim _{\varepsilon \rightarrow+0} v(x, t, \varepsilon),
$$

of the weak asymptotic solution $(u(x, t, \varepsilon), v(x, t, \varepsilon))$ to this Cauchy problem.

We will construct a weak asymptotic solution of the Cauchy problem as the sum of the singular ansatz regularized with respect to singularities $H(-x+\phi(t))$ and $\delta(-x+\phi(t))$, and corrections:

$$
\begin{aligned}
& u(x, t, \varepsilon)=\widetilde{u}(x, t, \varepsilon)+R_{u}(x, t, \varepsilon), \\
& v(x, t, \varepsilon)=\widetilde{v}(x, t, \varepsilon)+R_{v}(x, t, \varepsilon),
\end{aligned}
$$

where a pair of functions $(\widetilde{u}(x, t, \varepsilon), \widetilde{v}(x, t, \varepsilon))$ is a regularization of the singular ansatz (1.12), and the corrections $R_{u}(x, t, \varepsilon), R_{v}(x, t, \varepsilon)$ are the desired functions, which must admit the estimates:

$$
R_{j}(x, t, \varepsilon)=o_{\mathcal{D}^{\prime}}(1), \quad \frac{\partial R_{j}(x, t, \varepsilon)}{\partial t}=o_{\mathcal{D}^{\prime}}(1), \quad \varepsilon \rightarrow+0, \quad j=u, v .
$$

Let us note that choosing the corrections is an essential part of the "right" construction of the weak asymptotic solution [6]- [9], [23], [24] (see Remarks 3.1, 4.1, and Sec. 5).

We shall construct a regularization $f(x, \varepsilon)$ of the distribution $f(x) \in \mathcal{D}^{\prime}(\mathbb{R})$ as

$$
f(x, \varepsilon)=f(x) * \frac{1}{\varepsilon} \omega\left(\frac{x}{\varepsilon}\right), \quad \varepsilon>0,
$$

where $*$ is a convolution, and a mollifier $\omega(\eta)$ has the following properties: (a) $\omega(\eta) \in C^{\infty}(\mathbb{R})$, (b) $\omega(\eta)$ has a compact support or decreases sufficiently rapidly as $|\eta| \rightarrow \infty, \quad$ (c) $\int \omega(\eta) d \eta=1$, (d) $\omega(\eta) \geq 0, \quad($ e) $\omega(-\eta)=\omega(\eta)$. We have $\lim _{\varepsilon \rightarrow+0}\langle f(\xi, \varepsilon), \phi(\xi)\rangle=\langle f, \phi\rangle$ for all $\phi \in \mathcal{D}(\mathbb{R})$. 
Thus, we will seek a weak asymptotic solution in the form

$$
\begin{array}{r}
u(x, t, \varepsilon)=u_{0}(x, t)+u_{1}(x, t) H_{u}(-x+\phi(t), \varepsilon) \\
\quad+R_{u}(x, t, \varepsilon) \\
v(x, t, \varepsilon)=\begin{aligned}
v_{0}(x, t)+ & v_{1}(x, t) H_{v}(-x+\phi(t), \varepsilon) \\
& +e(t) \delta(-x+\phi(t), \varepsilon)+R_{v}(x, t, \varepsilon),
\end{aligned}
\end{array}
$$

where according to $(1.15)$,

$$
\delta(x, \varepsilon)=\frac{1}{\varepsilon} \omega_{\delta}(x / \varepsilon),
$$

is a regularization of the $\delta$-function, and

$$
H_{j}(x, \varepsilon)=\omega_{0 j}\left(\frac{x}{\varepsilon}\right)=\int_{-\infty}^{x / \varepsilon} \omega_{j}(\eta) d \eta, \quad j=u, v
$$

are regularizations of the Heaviside function $H(x)$. Here the mollifiers $\omega_{u}(\eta), \omega_{v}(\eta)$, $\omega_{\delta}(\eta)$ have properties $(\mathrm{a})-(\mathrm{e})$. It is clear that $\omega_{0 j}(\eta) \in C^{\infty}(\mathbb{R}), \lim _{\eta \rightarrow+\infty} \omega_{0 j}(\eta)=1$, $\lim _{\eta \rightarrow-\infty} \omega_{0 j}(\eta)=0, j=u, v$.

Let $\lambda_{1}(u, v), \lambda_{2}(u, v)$ be the eigenvalues of the characteristic matrix of system (1.1). As in [10], [13], [26], we use the "overcompression" condition

$$
\begin{aligned}
& \lambda_{1}\left(u_{+}, v_{+}\right) \leq \dot{\phi}(t) \leq \lambda_{1}\left(u_{-}, v_{-}\right) \\
& \lambda_{2}\left(u_{+}, v_{+}\right) \leq \dot{\phi}(t) \leq \lambda_{2}\left(u_{-}, v_{-}\right)
\end{aligned}
$$

as the admissibility condition for the $\delta$-shocks, where $\dot{\phi}(t)$ is the velocity of motion of the $\delta$-shock front, and $u_{-}=u_{0}+u_{1}, v_{-}=v_{0}+v_{1}$ and $u_{+}=u_{0}, v_{+}=v_{0}$ are the respective left- and right-hand values of $u, v$ on the discontinuity curve. It means that all characteristics on both sides of the discontinuity are in-coming.

\section{2. $\delta$-SHOCK WAVE TYPE SOLUTIONS}

2.1. Generalized solutions. Suppose that $\Gamma=\left\{\gamma_{i}: i \in I\right\}$ is a connected graph in the upper half-plane $\{(x, t): x \in \mathbb{R}, t \in[0, \infty)\} \in \mathbb{R}^{2}$ containing smooth arcs $\gamma_{i}$, $i \in I$, and $I$ is a finite set. By $I_{0}$ we denote a subset of $I$ such that an arc $\gamma_{k}$ for $k \in I_{0}$ starts from the points of the $x$-axis; $\Gamma_{0}=\left\{x_{k}^{0}: k \in I_{0}\right\}$ is the set of initial points of $\operatorname{arcs} \gamma_{k}, k \in I_{0}$.

Let $\left(u^{0}(x), v^{0}(x)\right)$ be $\delta$-shock wave type initial data, i.e.,

$$
v^{0}(x)=V^{0}(x)+e^{0} \delta\left(\Gamma_{0}\right)
$$

where $u^{0}, V^{0} \in L^{\infty}(\mathbb{R} ; \mathbb{R})$, and $e^{0} \delta\left(\Gamma_{0}\right) \stackrel{\text { def }}{=} \sum_{k \in I_{0}} e_{k}^{0} \delta\left(x-x_{k}^{0}\right), \quad e_{k}^{0}$ are constants, $k \in I_{0}$.

Let us introduce the definition of a $\delta$-shock wave type solution for system (1.1).

Definition 2.1. ( [7]- [9]) A pair of distributions $(u(x, t), v(x, t))$ and graph $\Gamma$, where $v(x, t)$ is represented in the form of the sum

$$
v(x, t)=V(x, t)+e(x, t) \delta(\Gamma)
$$

$u, V \in L^{\infty}(\mathbb{R} \times(0, \infty) ; \mathbb{R}), e(x, t) \delta(\Gamma) \stackrel{\text { def }}{=} \sum_{i \in I} e_{i}(x, t) \delta\left(\gamma_{i}\right), e_{i}(x, t) \in C^{1}(\Gamma), i \in I$, is called a generalized $\delta$-shock wave type solution of system (1.1) with the initial data 
$\left(u^{0}(x), v^{0}(x)\right)$ if the integral identities

$$
\begin{aligned}
\int_{0}^{\infty} \int\left(u \varphi_{t}+F(u, V) \varphi_{x}\right) d x d t+\int u^{0}(x) \varphi(x, 0) d x & =0 \\
\int_{0}^{\infty} \int\left(V \varphi_{t}+G(u, V) \varphi_{x}\right) d x d t+\sum_{i \in I} \int_{\gamma_{i}} e_{i}(x, t) \frac{\partial \varphi(x, t)}{\partial \mathbf{l}} d l & \\
+\int V^{0}(x) \varphi(x, 0) d x+\sum_{k \in I_{0}} e_{k}^{0} \varphi\left(x_{k}^{0}, 0\right) & =0
\end{aligned}
$$

hold for all test functions $\varphi(x, t) \in \mathcal{D}(\mathbb{R} \times[0, \infty))$, where $\frac{\partial \varphi(x, t)}{\partial \mathbf{l}}$ is the tangential derivative on the graph $\Gamma, \int_{\gamma_{i}} \cdot d l$ is a line integral over the arc $\gamma_{i}$.

\subsection{The Rankine-Hugoniot conditions.}

Theorem 2.1. Let us assume that $\Omega \subset \mathbb{R} \times(0, \infty)$ is some region cut by a smooth curve $\Gamma$ into a left- and right-hand parts $\Omega_{\mp},(u(x, t), v(x, t))$ and $\Gamma$ is a generalized $\delta$-shock wave type solution of system (1.1) and $(u(x, t), v(x, t))$ is smooth in $\Omega_{ \pm}$. Then the Rankine-Hugoniot conditions for $\delta$-shocks

$$
\begin{aligned}
& {[F(u, v)]_{\Gamma} \nu_{1}+[u]_{\Gamma} \nu_{2}=0,} \\
& {[G(u, v)]_{\Gamma} \nu_{1}+[v]_{\Gamma} \nu_{2}=\frac{\left.\partial e(x, t)\right|_{\Gamma}}{\partial \mathbf{l}},}
\end{aligned}
$$

hold along $\Gamma$, where $\mathbf{n}=\left(\nu_{1}, \nu_{2}\right)$ is the unit normal to the curve $\Gamma$ pointing from $\Omega_{-}$ into $\Omega_{+}, \mathbf{l}=\left(-\nu_{2}, \nu_{1}\right)$,

$$
\left.[h(u, v)]\right|_{\Gamma}=\left.\left(h\left(u_{-}, v_{-}\right)-h\left(u_{+}, v_{+}\right)\right)\right|_{\Gamma}
$$

is a jump in function $h(u(x, t), v(x, t))$ across the discontinuity curve $\Gamma,\left(u_{\mp}, v_{\mp}\right)$ are respective left- and right-hand values of $(u, v)$ on the discontinuity curve.

If $\Gamma=\{(x, t): x=\phi(t)\}, \Omega_{ \pm}=\{(x, t): \pm(x-\phi(t))>0\}$ then relations $(2.2)$ can be rewritten as

$$
\begin{aligned}
\dot{\phi}(t) & =\left.\frac{[F(u, v)]}{[u]}\right|_{x=\phi(t)}, \\
\dot{e}(t) & =\left.\left([G(u, v)]-[v] \frac{[F(u, v)]}{[u]}\right)\right|_{x=\phi(t)}
\end{aligned}
$$

where $\left.e(t) \stackrel{\text { def }}{=} e(x, t)\right|_{x=\phi(t)}$, and $(\cdot)=\frac{d}{d t}(\cdot)$.

Proof. Selecting the test function $\varphi(x, t)$ with compact support in $\Omega_{ \pm}$, we deduce from (2.1) that (1.1) hold in $\Omega_{ \pm}$, respectively. Now choosing a test function $\varphi(x, t)$ with support in $\Omega$, we deduce from the second identity (2.1) that

$$
\begin{aligned}
& 0=\int_{0}^{\infty} \int\left(V \varphi_{t}+G(u, V) \varphi_{x}\right) d x d t \\
= & \iint_{\Omega_{-}}\left(V \varphi_{t}+G(u, V) \varphi_{x}\right) d x d t+\iint_{\Omega_{+}}\left(V \varphi_{t}+G(u, V) \varphi_{x}\right) d x d t .
\end{aligned}
$$

Next, integrating by parts, we obtain

$$
\begin{aligned}
& \iint_{\Omega_{ \pm}}\left(V \varphi_{t}+G(u, V) \varphi_{x}\right) d x d t \\
&=-\iint_{\Omega_{ \pm}}\left(V_{t}+(G(u, V))_{x}\right) \varphi d x d t \mp \int_{\Gamma}\left(\nu_{2} v_{ \pm}+\nu_{1} G\left(u_{ \pm}, v_{ \pm}\right)\right) \varphi d l \\
&=\mp \int_{\Gamma}\left(\nu_{2} v_{ \pm}+\nu_{1} G\left(u_{ \pm}, v_{ \pm}\right)\right) \varphi d l
\end{aligned}
$$


owing to (1.1). Adding the last relations, we have

$$
\int_{0}^{\infty} \int\left(V \varphi_{t}+G(u, V) \varphi_{x}\right) d x d t=\int_{\Gamma}\left([G(u, v)] \nu_{1}+[v] \nu_{2}\right) \varphi(x, t) d l
$$

for all $\varphi(x, t) \in \mathcal{D}(\Omega)$.

Now integrating by parts we can easily see that

$$
\int_{\Gamma} e(x, t) \frac{\partial \varphi(x, t)}{\partial \mathbf{l}} d l=-\int_{\Gamma} \frac{\partial e(x, t)}{\partial \mathbf{l}} \varphi(x, t) d l
$$

where $\left.\frac{\partial}{\partial \mathbf{l}} e(x, t)\right|_{\Gamma}=\left.\frac{\partial}{\partial t} e(x, t)\right|_{\Gamma} \nu_{1}-\left.\frac{\partial}{\partial x} e(x, t)\right|_{\Gamma} \nu_{2}$.

Adding (2.4) and (2.5), we deduce

$$
\int_{\Gamma}\left([G(u, v)] \nu_{1}+[v] \nu_{2}-\frac{\partial e(x, t)}{\partial \mathbf{l}}\right) \varphi(x, t) d l=0
$$

for all $\varphi(x, t) \in \mathcal{D}(\Omega)$. Thus the second relation (2.2) holds.

We obtain the proof of the first relation (2.2) using formula (2.4).

$$
\begin{gathered}
\text { If } \Gamma=\{(x, t): x=\phi(t)\} \text { then } \mathbf{n}=\left(\nu_{1}, \nu_{2}\right)=\frac{(1,-\dot{\phi}(t))}{\sqrt{1+(\dot{\phi}(t))^{2}}}, \mathbf{l}=\frac{(\dot{\phi}(t), 1)}{\sqrt{1+(\dot{\phi}(t))^{2}}} \text {, and } \\
\frac{\left.\partial \varphi(x, t)\right|_{\Gamma}}{\partial \mathbf{l}}=\frac{1}{\sqrt{1+(\dot{\phi}(t))^{2}}} \frac{d \varphi(\phi(t), t)}{d t} .
\end{gathered}
$$

In view of (2.6), relations (2.2) imply (2.3).

The first equation (2.2) (or (2.3)) is the standard Rankine-Hugoniot condition. The left-hand side of the second equation (2.2) (or (2.3)) is called the RankineHugoniot deficit.

The system of $\delta$-shocks integral identities (2.1) is a natural generalization of the system of integral identities (1.2). The integral identities (2.1) differ from (1.2) by an additional term

$$
\int_{\Gamma} e(x, t) \frac{\partial \varphi(x, t)}{\partial \mathbf{l}} d l=\sum_{i \in I} \int_{\gamma_{i}} e_{i}(x, t) \frac{\partial \varphi(x, t)}{\partial \mathbf{l}} d l
$$

in the second identity. This term appears due to the Rankine-Hugoniot deficit.

2.3. Weak asymptotic solutions. Denote by $O_{\mathcal{D}^{\prime}}\left(\varepsilon^{\alpha}\right)$ the collection of distributions $f(x, t, \varepsilon) \in \mathcal{D}^{\prime}\left(\mathbb{R}_{x}\right)$ such that

$$
\langle f(x, t, \varepsilon), \psi(x)\rangle=O\left(\varepsilon^{\alpha}\right),
$$

for any test function $\psi(x) \in \mathcal{D}\left(\mathbb{R}_{x}\right)$. Moreover, $\langle f(x, t, \varepsilon), \psi(x)\rangle$ is a continuous function in $t$, where the estimate $O\left(\varepsilon^{\alpha}\right)$ is understood in the standard sense and is uniform with respect to $t$. The relation $o_{\mathcal{D}^{\prime}}\left(\varepsilon^{\alpha}\right)$ is understood in a corresponding way.

Definition 2.2. ( $[6]-[9])$ A pair of functions $(u(x, t, \varepsilon), v(x, t, \varepsilon))$ smooth as $\varepsilon>0$ is called a weak asymptotic solution of system (1.1) with the initial data $\left(u^{0}(x), v^{0}(x)\right)$ if

$$
\begin{aligned}
L_{1}[u(x, t, \varepsilon), v(x, t, \varepsilon)] & =o_{\mathcal{D}^{\prime}}(1), \\
L_{2}[u(x, t, \varepsilon), v(x, t, \varepsilon)] & =o_{\mathcal{D}^{\prime}}(1), \\
u(x, 0, \varepsilon) & =u^{0}(x)+o_{\mathcal{D}^{\prime}}(1), \\
v(x, 0, \varepsilon) & =v^{0}(x)+o_{\mathcal{D}^{\prime}}(1), \quad \varepsilon \rightarrow+0
\end{aligned}
$$

where the first two estimates are uniform in $t$. 
Constructing the weak asymptotic solution and multiplying the first two relations (2.7) by a test function $\varphi(x, t) \in \mathcal{D}(\mathbb{R} \times[0, \infty))$, integrating these relations by parts and then passing to the limit as $\varepsilon \rightarrow+0$, we will see that the pair of distributions (1.13) satisfy the integral identities (2.1).

\section{Propagation of $\delta$-Shocks in System $(1.9)$}

3.1. Weak asymptotic solution. Consider the Cauchy problem (1.9), (1.11). In this case the graph $\Gamma$ contains only one arc. Suppose this arc has the form $\Gamma=$ $\{(x, t): x=\phi(t)\}$, and hence $\left.e(x, t)\right|_{\Gamma}=e(t)$. The first step of our approach is to find a weak asymptotic solution of the Cauchy problem (1.9), (1.11).

The eigenvalues of the characteristic matrix of system (1.9) are

$$
\lambda_{1,2}(u)=\frac{1}{2}\left(f^{\prime}(u) \pm \sqrt{\left(f^{\prime}(u)\right)^{2}-4 g^{\prime}(u)}\right), \quad\left(f^{\prime}(u)\right)^{2} \geq 4 g^{\prime}(u) .
$$

We assume that the "overcompression" condition (1.19) is satisfied.

We will seek a $\delta$-shock wave type solution in the form (1.12) and a weak asymptotic solution in the form (1.16). Since the generalized $\delta$-shock wave type solution is defined as a weak limit (1.13) of (1.16), in view of the estimates (1.14), the corrections $R_{u}(x, t, \varepsilon), R_{v}(x, t, \varepsilon)$ do not make a contribution to the generalized solution of the problem. However, according to (3.9), (3.10), these terms make a contribution to the weak asymptotics of the superposition $f(u(x, t, \varepsilon))-v(x, t, \varepsilon)$ and $g(u(x, t, \varepsilon))$, and hence play an essential role in the construction of the generalized solution to the problem. Without introducing these terms, we cannot solve the Cauchy problem with arbitrary initial data and cannot construct the "right" singular superpositions (see Remarks 3.1).

Here we choose the corrections in the special form

$$
\begin{aligned}
R_{u}(x, t, \varepsilon)= & P(t) \frac{1}{\varepsilon^{1 / n}} \Omega_{P}\left(\frac{-x+\phi(t)}{\varepsilon}\right) \\
R_{v}(x, t, \varepsilon)= & 0,
\end{aligned}
$$

where $P(t), Q(t)$ are the desired functions, $\frac{1}{\varepsilon} \Omega_{P}^{n}(x / \varepsilon), \frac{1}{\varepsilon} \Omega_{Q}^{n+1}(x / \varepsilon)$ are regularizations (1.17) of the delta function, mollifiers $\Omega_{P}(\eta), \Omega_{Q}(\eta)$ have properties (a)-(c). Consequently, estimates (1.14) hold.

In addition to (3.1), we can choose mollifiers $\Omega_{P}(\eta), \Omega_{Q}(\eta)$ such that

$$
\begin{aligned}
\int \Omega_{P}^{k}(\eta) \Omega_{Q}^{n+1-k}(\eta) d \eta=0, & k=1,2, \ldots n+1, \\
\int \Omega_{Q}^{n+1}(\eta) d \eta \neq 0, &
\end{aligned}
$$

In particular, for system (1.8) $f(u)=u^{2}, g(u)=\frac{1}{3} u^{3}-u$ and relations (3.2) have the form $\int \Omega_{P}^{3}(\eta) d \eta=0, \int \Omega_{P}^{2}(\eta) \Omega_{Q}(\eta) d \eta=0, \int \Omega_{P}(\eta) \Omega_{Q}^{2}(\eta) d \eta=0$.

Theorem 3.1. Let

$$
\lambda_{+}\left(u_{0}^{0}(0)\right) \leq\left.\frac{\left[f\left(u^{0}\right)\right]-\left[v^{0}\right]}{\left[u^{0}\right]}\right|_{x=0} \leq \lambda_{-}\left(u_{0}^{0}(0)+u_{1}^{0}(0)\right),
$$


then there exists $T>0$ such that, for $t \in[0, T)$, the Cauchy problem (1.9), (1.11) has a weak asymptotic solution (1.16), (3.1), (3.2) if and only if

$$
\begin{aligned}
& L_{11}\left[u_{+}, v_{+}\right]=0, \quad x>\phi(t), \\
& L_{11}\left[u_{-}, v_{-}\right]=0, \quad x<\phi(t), \\
& L_{12}\left[u_{+}, v_{+}\right]=0, \quad x>\phi(t), \\
& L_{12}\left[u_{-}, v_{-}\right]=0, \quad x<\phi(t), \\
& \dot{\phi}(t)=\left.\frac{[f(u)]-[v]}{[u]}\right|_{x=\phi(t)}, \\
& \dot{e}(t)=\left.\left([g(u)]-[v] \frac{[f(u)]-[v]}{[u]}\right)\right|_{x=\phi(t)}, \\
& P(t)=\left\{\frac{e(t)}{a A_{n}}\right)^{1 / n},\left\{\frac { e ( t ) } { c B _ { n + 1 } } \left(\frac{[f(u)]-[v]}{[u]}-\frac{1}{A_{n}}\left(B_{n}+\right.\right.\right. \\
&\left.\left.\left.Q(t)=\left.(n+1) B_{n+1}\left(u_{0}+\frac{b}{a} u_{1}\right)\right|_{x=\phi(t)}\right)\right)\right\}^{1 /(n+1)},
\end{aligned}
$$

where $u_{+}=u_{0}, v_{+}=v_{0}, u_{-}=u_{0}+u_{1}, v_{-}=v_{0}+v_{1}$,

$$
a=\int \Omega_{P}^{n}(\eta) d \eta>0, \quad b=\int \omega_{0 u}(\eta) \Omega_{P}^{n}(\eta) d \eta, \quad c=\int \Omega_{Q}^{n+1}(\eta) d \eta \neq 0 .
$$

The initial data for system (3.4), (3.5) are defined from (1.11), and

$$
\begin{aligned}
e(0)= & e^{0}, \\
P(0)= & \left(\frac{e^{0}}{a A_{n}}\right)^{1 / n}, \\
Q(0)= & \left\{\frac { e ^ { 0 } } { c B _ { n + 1 } } \left(\frac{[f(u)]-[v]}{[u]}-\frac{1}{A_{n}}\left(B_{n}\right.\right.\right. \\
& \left.\left.\left.+(n+1)\left(u_{0}+\frac{b}{a} u_{1}\right) B_{n+1}\right)\right)\right\}\left.^{1 /(n+1)}\right|_{x=0} .
\end{aligned}
$$

Proof. With the help of (3.2), (3.6) and relations (6.1) from Lemma 6.1, we find the following weak asymptotics

$$
\begin{aligned}
R^{k}(x, t, \varepsilon) & =o_{\mathcal{D}^{\prime}}(1), \quad k \leq n-1, \\
R^{n}(x, t, \varepsilon) & =a P^{n}(t) \delta(-x+\phi(t))+o_{\mathcal{D}^{\prime}}(1), \\
R^{n+1}(x, t, \varepsilon) & =c Q^{n+1}(t) \delta(-x+\phi(t))+o_{\mathcal{D}^{\prime}}(1), \\
H(-x+\phi(t), \varepsilon) R^{n}(x, t, \varepsilon) & =b P^{n}(t) \delta(-x+\phi(t))+o_{\mathcal{D}^{\prime}}(1)
\end{aligned}
$$

where $a, b, c$ are defined by (3.6).

Using relations (6.1) from Lemma 6.1, one can calculate

$$
\begin{aligned}
& (u(x, t, \varepsilon))^{k}=u_{0}^{k}+\left(\left(u_{0}+u_{1}\right)^{k}-u_{0}^{k}\right) H(-x+\phi(t)) \\
& +o_{\mathcal{D}^{\prime}}(1), \quad k \leq n-1, \\
& (u(x, t, \varepsilon))^{n}=u_{0}^{n}+\left(\left(u_{0}+u_{1}\right)^{n}-u_{0}^{n}\right) H(-x+\phi(t)) \\
& +R^{n}(x, t, \varepsilon)+o_{\mathcal{D}^{\prime}}(1), \\
& (u(x, t, \varepsilon))^{n+1}=u_{0}^{n+1}+\left(\left(u_{0}+u_{1}\right)^{n+1}-u_{0}^{n+1}\right) H(-x+\phi(t)) \\
& +(n+1)\left(u_{0}+u_{1} H(-x+\phi(t), \varepsilon)\right) \\
& \times R^{n}(x, t, \varepsilon)+R^{n+1}(x, t, \varepsilon)+o_{\mathcal{D}^{\prime}}(1) \text {. }
\end{aligned}
$$

Taking into account relations (3.7), (3.8), we obtain

$$
\begin{gathered}
f(u(x, t, \varepsilon))=f\left(u_{0}\right)+\left(f\left(u_{0}+u_{1}\right)-f\left(u_{0}\right)\right) H(-x+\phi(t)) \\
+a A_{n} P^{n}(t) \delta(-x+\phi(t))+o_{\mathcal{D}^{\prime}}(1), \\
g(u(x, t, \varepsilon))=g\left(u_{0}\right)+\left(g\left(u_{0}+u_{1}\right)-g\left(u_{0}\right)\right) H(-x+\phi(t))
\end{gathered}
$$




$$
\begin{aligned}
& +\left\{a B_{n} P^{n}(t)+(n+1)\left(a u_{0}+b u_{1}\right) B_{n+1} P^{n}(t)\right. \\
& \left.+c B_{n+1} Q^{n+1}(t)\right\} \delta(-x+\phi(t))+o_{\mathcal{D}^{\prime}}(1), \quad \varepsilon \rightarrow+0 .
\end{aligned}
$$

Substituting the smooth ansatz (1.16) and relations (3.9), (3.10) into the left-hand side of system (1.9), we obtain, up to $o_{\mathcal{D}^{\prime}}(1)$, the following relations

$$
\begin{aligned}
& L_{11}[u(x, t, \varepsilon), v(x, t, \varepsilon)] \\
&=L_{11}\left[u_{0}, v_{0}\right]+\left\{\frac{\partial u_{1}}{\partial t}+\frac{\partial}{\partial x}[f(u)-v]\right\} H(-x+\phi(t)) \\
&+\{[u] \dot{\phi}(t)-[f(u)-v]\} \delta(-x+\phi(t)) \\
&+\left\{e(t)-a A_{n} P^{n}(t)\right\} \delta^{\prime}(-x+\phi(t))+o_{\mathcal{D}^{\prime}}(1), \\
& L_{12}[u(x, t, \varepsilon), v(x, t, \varepsilon)] \\
&=L_{22}\left[u_{0}, v_{0}\right]+\left\{\frac{\partial v_{1}}{\partial t}+\frac{\partial}{\partial x}[g(u)]\right\} H(-x+\phi(t)) \\
&=\{[v] \dot{\phi}(t)+\dot{e}(t)-[g(u)]\} \delta(-x+\phi(t)) \\
&+\left\{e(t) \dot{\phi}(t)-a B_{n} P^{n}(t)-(n+1)\left(a u_{0}+b u_{1}\right) B_{n+1} P^{n}(t)\right. \\
&\left.-c B_{n+1} Q^{n+1}(t)\right\} \delta^{\prime}(-x+\phi(t))+o_{\mathcal{D}^{\prime}}(1), \quad \varepsilon \rightarrow+0 .
\end{aligned}
$$

Here we take into account estimates (1.14).

Setting the left-hand side of (3.11), (3.12) equal to zero, we obtain the necessary and sufficient conditions for the first two equalities (2.7), i.e., systems (3.4), (3.5).

Consider the Cauchy problem

$$
\begin{aligned}
& L_{11}[u, V]=0, \quad u(x, 0)=u^{0}(x), \\
& L_{12}[u, V]=0, \quad V(x, 0)=V^{0}(x)=v_{0}^{0}(x)+v_{1}^{0}(x) H(-x),
\end{aligned}
$$

assuming that condition (3.3) holds. The last condition means that $\left(u^{0}(x), V^{0}(x)\right)$ is entropy initial data. According to [17, Ch.4.2.], we extend a pair of functions

$$
\begin{aligned}
\left(u_{+}^{0}(x)=u_{0}^{0}(x), V_{+}^{0}(x)=v_{0}^{0}(x)\right), & x \leq 0, \\
\left(u_{-}^{0}(x)=u_{0}^{0}(x)+u_{1}^{0}(x), V_{-}^{0}(x)=v_{0}^{0}(x)+v_{1}^{0}(x)\right), & x \geq 0,
\end{aligned}
$$

in a bounded $C^{1}$ fashion and continue to denote the extended pair of functions by $\left(u_{ \pm}^{0}(x), V_{ \pm}^{0}(x)\right)$. By $\left(u_{ \pm}(x, t), V_{ \pm}(x, t)\right)$ we denote the $C^{1}$ solutions of the problems

$$
\begin{array}{ll}
L_{11}[u, V]=0, & u_{ \pm}(x, 0)=u_{ \pm}^{0}(x), \\
L_{12}[u, V]=0, & V_{ \pm}(x, 0)=V_{ \pm}^{0}(x),
\end{array}
$$

which, according to [17, Ch.2.1.], exist for small enough time interval $\left[0, T_{1}\right]$. The pair $\left(u_{ \pm}(x, t), V_{ \pm}(x, t)\right)$ determines a two-sheeted covering of the plane $(x, t)$. Next, we define the function $x=\phi(t)$ as a solution of the problem

$$
\dot{\phi}(t)=\left.\frac{\left.f\left(u_{-}(x, t)\right)-f\left(u_{+}(x, t)\right)-V_{-}(x, t)\right)+V_{+}(x, t)}{u_{-}(x, t)-u_{+}(x, t)}\right|_{x=\phi(t)},
$$

$\phi(0)=0$. It is clear that there exists a unique function $\phi(t)$ for sufficiently short times $\left[0, T_{2}\right]$. Setting $T=\min \left(T_{1}, T_{2}\right)$, we define the shock solution by

$$
(u(x, t), V(x, t))= \begin{cases}\left(u_{+}(x, t), V_{+}(x, t)\right), & x>\phi(t), \\ \left(u_{-}(x, t), V_{-}(x, t)\right), & x<\phi(t) .\end{cases}
$$

Thus the first five equations of system (3.4) define a unique solution of the Cauchy problem (3.13) for $t \in[0, T)$. Solving this problem, we obtain $u(x, t), V(x, t), \phi(t)$. 
Then, substituting these functions into (3.4), (3.5), we obtain $e(t), v(x, t)=$ $V(x, t)+e(t) \delta(-x+\phi(t))$, and $P(t), Q(t)$. It is clear that mollifiers $\Omega_{P}(\eta), \Omega_{Q}(\eta)$ can be chosen to satisfy relations $(3.2)$.

3.2. $\delta$-Shock wave type solution. At the second step, using the weak asymptotic solution constructed by Theorem 3.1, we obtain a generalized solution of the Cauchy problem (1.9), (1.11).

Theorem 3.2. There exists $T>0$ given by Theorem 3.1 such that the Cauchy problem (1.9), (1.11), (3.3) for $t \in[0, T)$ has a unique generalized solution (1.12), which satisfies the integral identities (2.1):

$$
\begin{aligned}
\int_{0}^{T} \int\left(u \varphi_{t}+\right. & \left.(f(u)-V) \varphi_{x}\right) d x d t \\
& +\int u^{0}(x) \varphi(x, 0) d x=0, \\
\int_{0}^{T} \int\left(V \varphi_{t}+g(u) \varphi_{x}\right) d x d t & +\int V^{0}(x) \varphi(x, 0) d x \\
+\int_{\Gamma} e(x, t) \frac{\partial \varphi(x, t)}{\partial \mathbf{l}} d l+e^{0} \varphi(0,0) & =0,
\end{aligned}
$$

where $\Gamma=\{(x, t): x=\phi(t), t \in[0, T)\}$,

$$
\int_{\Gamma} e(x, t) \frac{\partial \varphi(x, t)}{\partial \mathbf{l}} d l=\int_{0}^{T} e(t) \frac{d \varphi(\phi(t), t)}{d t} d t
$$

$V(x, t)=v_{0}(x, t)+v_{1}(x, t) H(-x+\phi(t)), \frac{d \varphi(\phi(t), t)}{d t}=\varphi_{t}(\phi(t), t)+\dot{\phi}(t) \varphi_{x}(\phi(t), t)(s e e$ $(2.6))$, and functions $u_{k}(x, t), v_{k}(x, t), \phi(t), e(t)$ are defined by system $(3.4)$.

Proof. By Theorem 3.1 we have the following estimates:

$$
L_{11}[u(x, t, \varepsilon)]=o_{\mathcal{D}^{\prime}}(\varepsilon), \quad L_{12}[u(x, t, \varepsilon), v(x, t, \varepsilon)]=o_{\mathcal{D}^{\prime}}(\varepsilon) .
$$

Let us apply the left-hand and right-hand sides of these relations to an arbitrary test function $\varphi(x, t) \in \mathcal{D}(\mathbb{R} \times[0, T))$. Since for $\varepsilon>0$ the functions $u(x, t, \varepsilon), v(x, t, \varepsilon)$ are smooth, then integrating by parts, we obtain

$$
\begin{gathered}
\int_{0}^{T} \int\left(u(x, t, \varepsilon) \varphi_{t}(x, t)+(f(u(x, t, \varepsilon))-v(x, t, \varepsilon)) \varphi_{x}(x, t)\right) d x d t \\
+\int u(x, 0, \varepsilon) \varphi(x, 0) d x=o(1), \\
\int_{0}^{T} \int\left(v(x, t, \varepsilon) \varphi_{t}(x, t)+g(u(x, t, \varepsilon)) \varphi_{x}(x, t)\right) d x d t \\
+\int v(x, 0, \varepsilon) \varphi(x, 0) d x=o(1), \quad \varepsilon \rightarrow+0 .
\end{gathered}
$$

Passing to the limit as $\varepsilon \rightarrow+0$, and taking into account (1.16), (3.1), (3.9), (3.10), (3.5), and the fact that

$$
\begin{gathered}
\lim _{\varepsilon \rightarrow+0} \int_{0}^{T} \int_{-\infty}^{\infty} e(t) \delta(-x+\phi(t), \varepsilon) \varphi(x, t) d x d t=\int_{0}^{T} e(t) \varphi(\phi(t), t) d t, \\
\lim _{\varepsilon \rightarrow+0} \int_{-\infty}^{\infty} e(0) \delta(-x, \varepsilon) \varphi(x, 0) d x=e(0) \varphi(0,0),
\end{gathered}
$$

we obtain the integral identities (3.14). According to Theorem 3.1, system (3.4) has a unique solution. 
The fifth and sixth equations of systems (3.4) are the Rankine-Hugoniot conditions of $\delta$-shocks, and the right-hand side of the sixth equation is the RankineHugoniot deficit.

Corollary 3.1. For $t \in[0, \infty)$, the Cauchy problem $(1.9),(1.11),(3.3)\left(u_{k}^{0}, v_{k}^{0}\right.$, $k=1,2$ are constants ) has a unique generalized solution (1.12), where

$$
\begin{aligned}
\phi(t) & =\frac{[f(u)]-[v]}{[u]} t \\
e(t) & =e^{0}+\left([g(u)]-[v] \frac{[f(u)]-[v]}{[u]}\right) t .
\end{aligned}
$$

By Theorem 3.2 and Corollary 3.1 we can obtain a $\delta$-shock type solution of the Cauchy problem (1.8), (1.11).

Remark 3.1. To find a generalized solution of the Cauchy problem (1.9), (1.11) and (1.8), (1.11) we construct a weak asymptotic solution of problem (1.16), where the functions $u_{k}(x, t), v_{k}(x, t), \phi(t), e(t), k=0,1$ are determined by relations (3.4) and the functions $\omega_{0 u}(\eta), \Omega_{P}(\eta), \Omega_{Q}(\eta), P(t), Q(t)$ are determined by relations $(3.2)$, (3.5), (3.6).

In view of estimate (1.14) (see also formulas (5.6), (5.7) below), the generalized solution (1.12) of the Cauchy problem does not depend on correction functions $P(t)$, $Q(t)$. However, according to (3.5), without introducing the terms

$$
P(t) \frac{1}{\varepsilon^{1 / n}} \Omega_{P}\left(\frac{-x+\phi(t)}{\varepsilon}\right), \quad Q(t) \frac{1}{\varepsilon^{1 /(n+1)}} \Omega_{Q}\left(\frac{-x+\phi(t)}{\varepsilon}\right),
$$

we cannot solve the Cauchy problem which admits $\delta$-shocks. If we introduce only the first term, we cannot solve the Cauchy problem with an arbitrary initial data (1.11), but only with initial values determined by the relation

$$
\frac{[f(u)]-[v]}{[u]}=\frac{1}{A_{n}}\left(B_{n}+(n+1)\left(u_{0}+\frac{b}{a} u_{1}\right) B_{n+1}\right),
$$

where the constants $a, b$ are defined by (3.6). This is related to the fact that system (3.4), (3.15) is overdetermined.

Without introducing the corrections we cannot also construct the "right" singular superpositions (5.6), (5.7) in Sec. 5.

\section{Propagation of $\delta$-Shocks in System $(1.5)$}

Let us consider the Cauchy problem (1.5), (1.11), where $u_{1}^{0}(0)>0$. The eigenvalues of the characteristic matrix of system (1.5) are $\lambda_{1}(u)=f^{\prime}(u), \lambda_{2}(u)=g(u)$. We shall assume that

$$
f^{\prime \prime}(u)>0, \quad g^{\prime}(u)>0, \quad f^{\prime}(u) \leq g(u)
$$

i.e., the "overcompression" condition (1.19) is satisfied.

We will seek a $\delta$-shock wave type solution in the form (1.12), a weak asymptotic solution in the form (1.16), and choose corrections in the form

$$
R_{u}(x, t, \varepsilon)=0, \quad R_{v}(x, t, \varepsilon)=R(t) \frac{1}{\varepsilon} \Omega^{\prime \prime}\left(\frac{-x+\phi(t)}{\varepsilon}\right),
$$

where $R(t)$ is a continuous function, $\varepsilon^{-3} \Omega^{\prime \prime}(x / \varepsilon)$ is a regularization of the distribution $\delta^{\prime \prime}(x), \Omega(\eta)$ has the properties (a)-(c) (see Sec. 1). It is clear that estimates (1.14) hold.

In $[6]-[9]$ the following theorems were proved. 
Theorem 4.1. There exists $T>0$ such that, for $t \in[0, T)$, the Cauchy problem (1.5), (1.11), (4.1) has a weak asymptotic solution (1.16), (4.2) if and only if

$$
\begin{aligned}
L_{21}\left[u_{0}\right] & =0, \quad x>\phi(t), \\
L_{21}\left[u_{0}+u_{1}\right] & =0, \quad x<\phi(t), \\
L_{22}\left[u_{0}, v_{0}\right] & =0, \quad x>\phi(t), \\
L_{22}\left[u_{0}+u_{1}, v_{0}+v_{1}\right] & =0, \quad x<\phi(t), \\
\dot{\phi}(t) & =\left.\frac{[f(u)]}{[u]}\right|_{x=\phi(t)}, \\
\dot{e}(t) & =\left.\left([v g(u)]-[v] \frac{[f(u)]}{[u]}\right)\right|_{x=\phi(t)}, \\
R(t)=\frac{e(t)}{c(t)} & \left(\left.\frac{[f(u)]}{[u]}\right|_{x=\phi(t)}-a(t)\right),
\end{aligned}
$$

where

$$
\begin{aligned}
a(t) & =\left.\int g\left(u_{-}(x, t) \omega_{0 u}(\eta)+u_{+}(x, t)\left(1-\omega_{0 u}(\eta)\right)\right)\right|_{x=\phi(t)} \omega_{\delta}(\eta) d \eta, \\
c(t) & =\left.\int g\left(u_{-}(x, t) \omega_{0 u}(\eta)+u_{+}(x, t)\left(1-\omega_{0 u}(\eta)\right)\right)\right|_{x=\phi(t)} \Omega^{\prime \prime}(\eta) d \eta \neq 0,
\end{aligned}
$$

$u_{-}=u_{0}+u_{1}, v_{-}=v_{0}+v_{1}, u_{+}=u_{0}, v_{+}=v_{0}$. The initial data for system (4.3), (4.4) are defined from (1.11), and

$$
\phi(0)=0, \quad R(0)=\frac{e^{0}}{c(0)}\left(\left.\frac{\left[f\left(u^{0}\right)\right]}{\left[u^{0}\right]}\right|_{x=0}-a(0)\right) .
$$

In [6]- [9], to prove Theorem 4.1 we use the weak asymptotics $v(x, t, \varepsilon) g(u(x, t, \varepsilon))$, $f(u(x, t, \varepsilon))$ given by Lemma 6.2 .

Theorem 4.2. Assume that conditions (4.1) are satisfied. Then, for $t \in[0, T)$, where $T>0$ is given by Theorem 4.1, the Cauchy problem (1.5), (1.11), has a unique generalized solution (1.12), which satisfies the integral identities (2.1):

$$
\begin{aligned}
\int_{0}^{T} \int\left(u \varphi_{t}+f(u) \varphi_{x}\right) d x d t+\int u^{0}(x) \varphi(x, 0) d x & =0 \\
\int_{0}^{T} \int\left(\varphi_{t}+g(u) \varphi_{x}\right) V d x d t+\int V^{0}(x) \varphi(x, 0) d x & \\
\int_{\Gamma} e(x, t) \frac{\partial \varphi(x, t)}{\partial \mathbf{l}} d l+e^{0} \varphi(0,0) & =0
\end{aligned}
$$

for all $\varphi(x, t) \in \mathcal{D}(\mathbb{R} \times[0, T))$, where $\Gamma=\{(x, t): x=\phi(t), \quad t \in[0, T)\}$,

$$
\int_{\Gamma} e(x, t) \frac{\partial \varphi(x, t)}{\partial \mathbf{l}} d l=\int_{0}^{T} e(t)\left(\varphi_{t}(\phi(t), t)+\dot{\phi}(t) \varphi_{x}(\phi(t), t)\right) d t,
$$

$V(x, t)=v_{0}+v_{1} H(-x+\phi(t))$. Here functions $u_{k}(x, t), v_{k}(x, t), k=0,1, \phi(t), e(t)$ are defined by system (4.3) with the initial data defined from (1.11), $\phi(0)=0$.

Corollary 4.1. For $t \in[0, \infty)$, the Cauchy problem (1.5), (1.11) $\left(u_{k}^{0}, v_{k}^{0}, k=1,2\right.$ are constants ) has a unique generalized solution (1.12), where

$$
\begin{aligned}
\phi(t) & =\frac{[f(u)]}{[u]} t \\
e(t) & =e^{0}+\left([g(u) v]-\frac{[f(u)]}{[u]}[v]\right) t .
\end{aligned}
$$

Remark 4.1. According to (4.4), (4.5), without introducing the corrections (4.2) we can only solve the Cauchy problem with initial data determined by the relation

$$
\left.\frac{[f(u(x, t))]}{[u(x, t)]}\right|_{x=\phi(t)}=\int g\left(u_{0}(\phi(t), t)+u_{1}(\phi(t), t) \omega_{0 u}(\eta)\right) \omega_{\delta}(\eta) d \eta .
$$


In this case we cannot construct the "right" singular superpositions (5.8), (5.9) defined in Sec. 5 .

\section{Singular Superpositions (Products) OF Distributions}

5.1. Singular superpositions. It seems natural to introduce the product of the Heaviside function and delta function as the weak limit of the product of their regularizations. Then, according to the second relation (6.1), we have

$$
\overbrace{H(x) \delta(x)}^{\text {def }} \lim _{\varepsilon \rightarrow+0} H(x, \varepsilon) \delta(x, \varepsilon)=B_{1} \delta(x),
$$

where $B_{1}=\int \omega_{0}(\eta) \omega_{\delta}(\eta) d \eta$. The product (5.1) defined in this way depends on the mollifiers $\omega, \omega_{\delta}$, i.e., on the regularizations of distributions $H(x), \delta(x)$.

In a similar way, we can introduce the singular superpositions $f(u(x, t))-v(x, t)$, $g(u(x, t))$, where distributions $u(x, t), v(x, t)$ are given by (1.12) and polynomials $f(u), g(u)$ are given by (1.10). Using regularizations of distributions (1.12) $u(x, t, \varepsilon)$, $v(x, t, \varepsilon)$ given by (1.16), (3.1), (3.2) and weak asymptotics (3.9), (3.10), we define singular superpositions by the following definition:

$$
\begin{gathered}
\overbrace{f(u(x, t))-v(x, t) \stackrel{\text { def }}{=}} \lim _{\varepsilon \rightarrow+0}(f(u(x, t, \varepsilon))-v(x, t, \varepsilon))=f\left(u_{0}\right)-v_{0} \\
+[f(u)-v] H(-x+\phi(t))+\left\{a A_{n} P^{n}(t)-e(t)\right\} \delta(-x+\phi(t)), \\
\overbrace{g(u(x, t))}^{\text {def }}=\lim _{\varepsilon \rightarrow+0} g(u(x, t, \varepsilon))=g\left(u_{0}\right)+[g(u)] H(-x+\phi(t)) \\
+\left\{a B_{n} P^{n}(t)+(n+1)\left(a u_{0}+b u_{1}\right) B_{n+1} P^{n}(t)+c B_{n+1} Q^{n+1}(t)\right\} \delta(-x+\phi(t)) .
\end{gathered}
$$

where the correction functions $P(t), Q(t)$ are given by (3.5), and $a, b, c$ by (3.6), and the limits are understood in the weak sense.

Let $f(u), g(u)$ be smooth functions. In the same way, using regularizations of distributions (1.12) $u(x, t, \varepsilon), v(x, t, \varepsilon)$ given by (1.16), (4.2) and weak asymptotics given by Lemma 6.2, we define the singular superpositions:

$$
\begin{aligned}
& \overbrace{f(u(x, t))} \stackrel{\text { def }}{=} \lim _{\varepsilon \rightarrow+0} f(u(x, t, \varepsilon))=f\left(u_{0}\right)+[f(u)] H(-x+\phi(t)), \\
& \quad+[g(u) v] H(-x+\phi(t))+\{e(t) a(t)+R(t) c(t)\} \delta(-x+\phi(t)),
\end{aligned}
$$

where $a(t), c(t)$ are defined by (4.5).

It is easy to see that the singular superpositions (5.2)-(5.5) depend on the regularizations of the Heaviside function, delta function and the correction functions $P(t), Q(t), R(t)$. This fact means that the above introduced singular superpositions are not unique.

5.2. "Right" singular superpositions. However, in the context constructing of weak asymptotic solutions of the Cauchy problems we can define explicit formulas for the "right" singular superpositions.

Namely, substituting $P(t), Q(t)$ given by (3.5) into expressions (5.2), (5.3), we obtain "right" unique singular superpositions:

$$
\begin{aligned}
f(u(x, t))-v(x, t) & \stackrel{\text { def }}{=} \lim _{\varepsilon \rightarrow+0}(f(u(x, t, \varepsilon))-v(x, t, \varepsilon)) \\
& =f\left(u_{0}\right)-v_{0}+[f(u)-v] H(-x+\phi(t)),
\end{aligned}
$$




$$
\begin{aligned}
& g(u(x, t)) \stackrel{\text { def }}{=} \lim _{\varepsilon \rightarrow+0}(g(u(x, t, \varepsilon))) \\
& \quad=g\left(u_{0}\right)+[g(u)] H(-x+\phi(t))+e(t) \frac{[f(u)]}{[u]} \delta(-x+\phi(t)) .
\end{aligned}
$$

Substituting $R(t)$ given by (4.4) into expressions (5.4), (5.5), we obtain "right" unique singular superpositions:

$$
\begin{aligned}
& f(u(x, t)) \stackrel{\text { def }}{=} \lim _{\varepsilon \rightarrow+0} f(u(x, t, \varepsilon))=f\left(u_{0}\right)+[f(u)] H(-x+\phi(t)), \\
& v(x, t) g(u(x, t)) \stackrel{\text { def }}{=} \lim _{\varepsilon \rightarrow+0} v(x, t, \varepsilon) g(u(x, t, \varepsilon))=v_{0} g\left(u_{0}\right) \\
& \quad+[v g(u)] H(-x+\phi(t))+e(t) \frac{[f(u)]}{[u]} \delta(-x+\phi(t)) .
\end{aligned}
$$

In (5.6)-(5.9) the distributions $u(x, t), v(x, t)$ are defined by (1.12) .

In contrast to $(5.2)-(5.5)$, where $u(x, t, \varepsilon), v(x, t, \varepsilon)$ are regularizations of distributions (1.12), in (5.6), (5.7), and (5.8), (5.9), u(x,t, $\varepsilon), v(x, t, \varepsilon)$ give the weak asymptotic solution of the Cauchy problem (1.9), (1.11), and (1.5), (1.11), respectively.

It is clear that the unique "right" singular superpositions (5.6)-(5.9) are independent of the regularizations of the Heaviside function, delta function and the correction functions and can be obtained only by the construction of a weak asymptotic solution of the Cauchy problem.

In fact, by (5.9) we define the unique "right" product of the Heaviside function and the delta function in the context of the Cauchy problem (1.5), (1.11). Setting $R(t)=0$ and comparing formulas (5.5) and (5.9), we readily see that to construct unique "right" product we must choose the mollifiers $\omega_{u}, \omega_{\delta}$ in (4.5) such that

$$
\begin{aligned}
a(t)=\left.\int g\left(u_{-}(x, t) \omega_{0 u}(\eta)+u_{+}(x, t)\left(1-\omega_{0 u}(\eta)\right)\right)\right|_{x=\phi(t)} \omega_{\delta}(\eta) d \eta & \\
& =\left.\frac{[f(u)]}{[u]}\right|_{x=\phi(t)} .
\end{aligned}
$$

In particular, for system (1.7) (here $f(u)=u^{2}, g(u)=u$ ) the unique "right" product of the Heaviside function and the delta function is defined as

$$
\begin{aligned}
e(t) \delta(-x+\phi(t)) u(x, t) & \\
=e(t) \delta(-x+\phi(t)) & \left\{\begin{array}{ll}
u_{-}(x, t), & x<\phi(t), \\
u_{+}(x, t), & x>\phi(t),
\end{array}=\left(u_{-}(x, t)+u_{+}(x, t)\right) e(t) \delta(-x+\phi(t)) .\right.
\end{aligned}
$$

Here according to (5.10), the mollifiers are such that

$$
\int \omega_{0 u}(\eta) \omega_{\delta}(\eta) d \eta=\left.\frac{u_{-}(x, t)}{u_{-}(x, t)-u_{+}(x, t)}\right|_{x=\phi(t)},
$$

and according to (1.19), (4.1), $u_{+}(\phi(t), t) \leq 0$.

As was already mentioned above, systems (1.9) and (1.8) have a specific "strange" property and, in contrast to system (1.5), formulas (5.6), (5.7) do not define (!) the product of the Heaviside function and the $\delta$-function. Moreover, although (according to $(1.12)), u(x, t)$ does not depend (!) on the term $e(t) \delta(-x+\phi(t))$, the righthand side of the "right" singular superposition (5.7) does depend (!) on this term. Thus one can say that the term $e(t) \delta(-x+\phi(t))$ "appears in (5.7) from nothing". Analogously, the left-hand side in (5.6) depends on $e(t) \delta(-x+\phi(t))$, but the righthand side in (5.6) does not depend on this term. 
Thus a "right" singular superposition is determined only in the context of solving the Cauchy problem. If we knew the "right" singular superpositions (5.6), (5.7) and (5.8), (5.9) in advance then Theorem 3.2 and Theorem 4.2 could be proved explicitly by substituting these superpositions into (1.9), (3.14) and (1.5), (4.6), respectively.

\section{SOME WEAK ASYMPTOTIC EXPANSIONS}

In order to find a weak asymptotic solution of the Cauchy problems (1.9), (1.11) and $(1.5),(1.11)$, we need weak asymptotics calculated in the following lemmas.

Lemma 6.1. Let $\delta(x, \varepsilon)=\frac{1}{\varepsilon} \omega_{\delta}\left(\frac{x}{\varepsilon}\right), \frac{1}{\varepsilon} \Omega\left(\frac{x}{\varepsilon}\right)$ be regularizations (1.17) of the delta function, and $H(\xi, \varepsilon)=\omega_{0}\left(\frac{\xi}{\varepsilon}\right)=\int_{-\infty}^{\frac{x}{\varepsilon}} \omega(\eta) d \eta$, be regularization (1.18) of the Heaviside function $H(x), x \in \mathbb{R}$. Then

$$
\begin{aligned}
(H(\xi, \varepsilon))^{r} & =H(\xi)+O_{\mathcal{D}^{\prime}}(\varepsilon), \\
(H(x, \varepsilon))^{r} \delta(x, \varepsilon) & =B_{r} \delta(x)+O_{\mathcal{D}^{\prime}}(\varepsilon), \\
\delta(x, \varepsilon)\left(\omega\left(\frac{x}{\varepsilon}\right)\right)^{r} & =A_{r} \delta(x)+O_{\mathcal{D}^{\prime}}(\varepsilon), \quad \varepsilon \rightarrow+0,
\end{aligned}
$$

where $B_{r}=\int \omega_{0}^{r}(\eta) \omega_{\delta}(\eta) d \eta, A_{r}=\int \omega_{\delta}(\eta) \Omega^{r}(\eta) d \eta, \quad r=1,2, \ldots$

Proof. From (1.18), we obviously have the first relation in (6.1). Making the change of variables $x=\varepsilon \eta$, we obtain

$$
\begin{aligned}
& \left\langle\frac{1}{\varepsilon} \omega_{\delta}\left(\frac{x}{\varepsilon}\right)\left(\omega_{0}\left(\frac{x}{\varepsilon}\right)\right)^{r}, \psi(x)\right\rangle \\
& \quad=\int \omega_{0}^{r}(\eta) \omega_{\delta}(\eta) \psi(\varepsilon \eta) d \eta=B_{r} \psi(0)+O(\varepsilon), \quad \varepsilon \rightarrow+0,
\end{aligned}
$$

for all $\psi(x) \in \mathcal{D}(\mathbb{R})$, i.e., the second relation is proved. Since $\omega_{\delta}(\eta) \Omega^{r}(\eta)$ decreases sufficiently rapidly as $|\eta| \rightarrow \infty$, then following the same reasoning, we prove the third relation:

$$
\begin{aligned}
\left\langle\frac{1}{\varepsilon} \omega_{\delta}\left(\frac{x}{\varepsilon}\right)\left(\Omega\left(\frac{x}{\varepsilon}\right)\right)^{r}, \psi(x)\right\rangle & =\int \omega_{\delta}(\eta) \Omega^{r}(\eta) \psi(\varepsilon \eta) d \eta \\
=A_{r} \psi(0)+O(\varepsilon), \quad \varepsilon & \rightarrow+0, \quad \forall \psi(x) \in \mathcal{D}(\mathbb{R}), \quad r=1,2, \ldots
\end{aligned}
$$

Lemma 6.2. ( [5, Corollary 1.1.], [6]- [8]) If $f(u), g(u)$ are smooth functions, and $u(x, t, \varepsilon), v(x, t, \varepsilon)$ are defined by $(1.16)$, (4.2) then

$$
\begin{aligned}
& f(u(x, t, \varepsilon))=f\left(u_{0}\right)+[f(u)] H(-x+\phi(t))+O_{\mathcal{D}^{\prime}}(\varepsilon), \quad \varepsilon \rightarrow+0, \\
& v(x, t, \varepsilon) g(u(x, t, \varepsilon))=g\left(u_{0}\right) v_{0}+[g(u) v] H(-x+\phi(t)) \\
& \quad+\{e(t) a(t)+R(t) c(t)\} \delta(-x+\phi(t))+O_{\mathcal{D}^{\prime}}(\varepsilon), \quad \varepsilon \rightarrow+0,
\end{aligned}
$$

where $a(t), c(t)$ are defined by (4.5).

\section{REFERENCES}

[1] F. Bouchut, On zero pressure gas dynamics, Advances in Math. for Appl. Sci., World Scientific, 22, (1994), 171-190.

[2] V. G. Danilov, V. P. Maslov, V. M. Shelkovich, Algebra of singularities of singular solutions to first-order quasilinear strictly hyperbolic systems, Theor. Math. Phys., 114, no. 1, (1998), $1-42$.

[3] V. G. Danilov, G. A. Omel'yanov, V. M. Shelkovich, Weak Asymptotics Method and Interaction of Nonlinear Waves, in Mikhail Karasev (ed.), "Asymptotic Methods for Wave and Quantum Problems", Amer. Math. Soc. Transl., Ser. 2, 208, 2003, 33-165. 
[4] V. G. Danilov, V. M. Shelkovich, Propagation and interaction of nonlinear waves to quasilinear equations, Hyperbolic problems: Theory, Numerics, Applications (Eighth International Conference in Magdeburg, February/March 2000, v.I). International Series of Numerical Mathematics, v. 140, Birkhäuser Verlag Basel/Switzerland, 2001, 267-276.

[5] V. G. Danilov and V. M. Shelkovich, Propagation and interaction of shock waves of quasilinear equation, Nonlinear Studies, 8, no. 1, (2001), 135-169.

[6] V. G. Danilov, V. M. Shelkovich, Propagation and interaction of delta-shock waves of a hyperbolic system of conservation laws, In Hou, Thomas Y.; Tadmor, Eitan (Eds.), Hyperbolic Problems: Theory, Numerics, Applications. Proceedings of the Ninth International Conference on Hyperbolic Problems held in CalTech, Pasadena, March 25-29, 2002, Springer Verlag, 2003, 483-492.

[7] V. G. Danilov, V. M. Shelkovich, Delta-shock wave type solution of hyperbolic systems of conservation laws, pp.27, Preprint 2003-052 at the url: http://www.math.ntnu.no/conservation/2003/052.html

[8] V. G. Danilov, V. M. Shelkovich, Dynamics of propagation and interaction of delta-shock waves in conservation law systems, pp.40, Preprint 2003-068 at the url: http://www.math.ntnu.no/conservation/2003/068.html (to be published in Journal of Differential Equations)

[9] V. G. Danilov, V. M. Shelkovich, Propagation and interaction of $\delta$-shock waves to hyperbolic systems of conservation laws, Dokl. Ross. Akad. Nauk, 394, no. 1, (2004), 10-14. English transl. in Russian Doklady Mathematics., 69, no. 1, (2004).

[10] G. Ercole, Delta-shock waves as self-similar viscosity limits, Quart. Appl. Math., LVIII, no. 1, (2000), 177-199.

[11] Feiming Huang, Existence and uniqueness of discontinuous solutions for a class nonstrictly hyperbolic systems, In Chen, Gui-Qiang (ed.) et al. Advances in nonlinear partial differential equations and related areas. Proceeding of conf. dedicated to prof. Xiaqi Ding, China, 1997, $187-208$

[12] B. L. Keyfitz, Conservation laws, delta-shocks and singular shocks, In M. Grosser, G. Hormann and M. Oberguggenberger (Eds.), Nonlinear Theory of Generalized Functions, Chapman \& Hall/CRC, 1999, 99-112.

[13] B. Lee Keyfitz and H. C. Kranzer, Spaces of weighted measures for conservation laws with singular shock solutions, Journal of Differential Equations, 118, (1995), 420-451.

[14] Philippe Le Floch, An existence and uniqueness result for two nonstrictly hyperbolic systems, Nonlinear evolution equations that change type, Springer-Verlag, 1990, 126-138.

[15] Philippe Le Floch, Entropy weak solutions to nonlinear hyperbolic systems under nonconservative form, Commun. in partial differential equations, 13, no. 6, (1988), 669-727.

[16] Philippe Le Floch, Tai-Ping Liu, Existense theory for nonlinear hyperbolic systems in nonconservative form, Forum Math., 5, (1993), no. 3, 261-280.

[17] A. Majda, Compressible fluid flow and systems of conservation laws in several space variables, Springer-Verlag New York, Berlin, Heidelberg, Tokyo, 1984.

[18] V. P. Maslov, Three algebras corresponding to nonsmooth solutions of systems of quasilinear hyperbolic equations, Uspekhi Mat. Nauk, 35, (1980), no. 2, 252-253. (Russian).

[19] V. P. Maslov, Non-standard characteristics in asymptotical problems, In: Proceeding of the International Congress of Mathematicians, August 16-24, 1983, Warszawa, vol. I, AmsterdamNew York-Oxford: North-Holland, 1984, 139-185.

[20] G. Dal Maso, P. G. Le Floch, and F. Murat, Definition and weak stability of nonconservative products, J. Math. Pures Appl., 74, (1995), 483-548.

[21] S. Schecter, Existence of Dafermos profiles for singular shocks, Preprint 2003-051 at the url: http://www.math.ntnu.no/conservation/2003/051.html

[22] M. Sever, Viscous structure of singular shocks, Nonlinearity, 15, (2002), 705-725.

[23] V. M. Shelkovich, Delta-shock waves of a class of hyperbolic systems of conservation laws, in A. Abramian, S. Vakulenko, V. Volpert (Eds.), "Patterns and Waves", St. Petersburg, 2003, $155-168$.

[24] V. M. Shelkovich, A specific hyperbolic system of conservation laws admitting delta-shock wave type solutions, pp.29, Preprint 2003-059 at the url: http://www.math.ntnu.no/conservation/2003/059.html

[25] Wancheg Shen, Tong Zhang, The Riemann problem for the transportaion equations in gas dynamics, Memoirs of the Amer. Math. Soc., 137, no. 654, (1999), 1-77.

[26] Dechun Tan, Tong Zhang and Yuxi Zheng, Delta-shock waves as limits of vanishing viscosity for hyperbolic systems of conservation laws, Journal of Differential Equations, 112, (1994), $1-32$. 
[27] A. I. Volpert, The space BV and quasilinear equations, Math. USSR Sb., 2, (1967), 225-267.

Department of Mathematics, St.-Petersburg State Architecture and Civil Engineering University, 2 Krasnoarmeiskaya 4, 190005, St. Petersburg, Russia.

E-mail address: shelkv@VS1567.spb.edu 